\title{
The effective action of a BPS Alice string
}

\author{
Chandrasekhar Chatterjee ${ }^{\mathrm{a}}$, Muneto Nitta \\ Department of Physics, and Research and Education Center for Natural Sciences, Keio University, Hiyoshi 4-1-1, Yokohama, Kanagawa \\ 223-8521, Japan
}

Received: 10 October 2017 / Accepted: 31 October 2017 / Published online: 28 November 2017

(C) The Author(s) 2017. This article is an open access publication

\begin{abstract}
Recently a BPS Alice string has been found in a $U(1) \times S O$ (3) gauge theory coupled with three charged complex scalar fields in the triplet representation (in JHEP 1709:046 arXiv:1703.08971 [hep-th], 2017). It is a half BPS state preserving a half of the supercharges when embedded into a supersymmetric gauge theory. In this paper, we study zero modes of a BPS Alice string. After presenting $U(1)$ and translational zero modes, we construct the effective action of these modes. In contrast to a previous analysis of the conventional Alice string for which only large distance behaviors are known, we can exactly perform a calculation in the full space thanks to the BPS properties.
\end{abstract}

\section{Introduction}

Topological vortices are an important and interesting subject to study not only because of their mathematical elegance but also as regards practical purposes from superfluids, superconductors, ultracold atomic gases to quantum field theory, QCD, string theory and cosmology. In a cosmological context, a symmetry breaking phase transition may have occurred in the early universe due to rapid cooling and expansion of universe, during which topological vortices known as 'cosmic strings' may be created [2,3]. Such classical vortex configurations become more interesting when interacting with quantum fields and these interactions generate massless excitations (zero modes) near the vortex core and dictate the low-energy dynamics of vortices. When a $U(1)$ zero mode arises due to a breaking of bulk continuous symmetry inside the vortex core, the string core behaves as a superconducting wire [4]. In the case of a local symmetry the excitation of a zero mode inside the vortex may also excite massless gauge field in the bulk which generates logarithmically divergent energy.

\footnotetext{
a e-mail: chandra@phys-h.keio.ac.jp

be-mail: nitta@phys-h.keio.ac.jp
}

Alice strings have very peculiar features. When a charged particle encircles an Alice string, its electric charge changes sign [5], and therefore one cannot define an electric charge globally. The simplest example of an Alice string can be found in an $S O$ (3) gauge theory with the 5 representation, in which the $S O(3)$ gauge group is spontaneously broken down to $O(2)$ in the vacuum. So the vacuum manifold or order parameter space becomes $G / H=S O(3) / O(2) \simeq \mathbb{R} P^{2}$. This allows for a non-trivial homotopy group $\pi_{1}\left(\mathbb{R} P^{2}\right) \simeq \mathbb{Z}_{2}$ supporting an Alice string. The unbroken generator can be identified as the electromagnetic $U(1)$ generator, and its sign changes while it encircles the Alice string once, as mentioned above. The bulk system of the Alice string behaves as 'Alice electrodynamics' where charge conjugation $\left(\mathbb{Z}_{2}\right)$ is taken as a local symmetry [6]. A change of the sign of the electric charges around the Alice string implies charge loss. This loss can be explained in terms of a nonlocal charge called the "Cheshire charge" [7-9]. ${ }^{1}$ Many interesting global phenomena arise due to this exotic property, like the generalized Aharonov-Bohm effect [14], anyonic exchange statistics etc. [15]. It has been suggested that the nature of the Cheshire charge can be explained as follows.

The $U(1)$ gauge symmetry in the bulk is spontaneously broken in the presence of an Alice string, thereby giving rise to a $U(1)$ zero mode, in addition to the usual translational zero modes. This $U(1)$ zero mode is non-normalizable and is charged under the $U(1)$ gauge group. If there are two or more Alice strings, the Cheshire charge should be nonlocally defined over them in terms of a zero mode attached to each string. However, the unbroken bulk symmetries are multivalued in the case of Alice strings, and therefore the behavior of the zero mode analysis becomes a little subtle, in which

\footnotetext{
1 The Alice string is also known to exchange magnetic charges from magnetic particles which creates the magnetic Cheshire charges [9]. Related to this, a ring of the Alice string can be thought of as a magnetic monopole at large distances when the $U$ (1) modulus is twisted along the ring, and this is supported by the non-trivial second homotopy group, $\pi_{2}(G / H) \neq 0$ [10-13].
} 
case one needs to carefully take into account the multivalued nature of the unbroken group generator in the analysis, which is called "obstruction" [16-19]. ${ }^{2}$ In general for vortices, this occurs due to the existence of local discrete unbroken symmetries [20].

One of the difficulties to perform a concrete calculation of a zero mode analysis in a multi-string system may be due to the existence of the interaction between Alice strings. The homotopy group $\pi_{1}\left(\mathbb{R} P^{2}\right) \simeq \mathbb{Z}_{2}$, implying that two Alice strings can annihilate each other and the existence of an attraction between them. One usually manipulates for instance an exchange of strings as an adiabatic process but it is dynamically difficult or impossible. In contrast, there is no force between Bogomolnyi-PrasadSommerfield (BPS) solitons (strings) [24, 25]. Therefore, one can place them at arbitrary positions, and $n$ strings have a total energy (tension) exactly $n$ times larger than that of a single string, thereby allowing for a large moduli space of multi-string configurations. However, in contrast to the conventional strings in the Abelian Higgs model [26,27], which can become BPS at the critical coupling, previously studied, Alice strings were all non-BPS because two Alice strings annihilate each other as mentioned above. In our recent work [1], we have found that an $U(1) \times S O(3)$ gauge theory coupled with three complex scalar fields in the triplet representation admits BPS Alice strings, and we have shown that it preserves a half supersymmetry if embedded into supersymmetric gauge theory. In this theory, one can expect to be able to place as many Alice strings as one likes at arbitrary positions, thereby opening the possibility to study the exotic phenomena of Alice strings more concretely.

In the present paper, as a first step, we systematically analyze the zero mode of a single BPS Alice string. A zero mode analysis for a conventional Alice string has been already discussed in the literature but only at large distance, since the equations cannot be solved exactly. In this work we shall show that the zero modes of a BPS Alice string can be solved exactly as functions of the vortex profile functions, so that the behaviors of the zero modes can be understood on the full space. We then construct the effective action for the $U(1)$ and translational zero modes of a single BPS Alice string.

This paper is organized as follows. In Sect. 2, we give a brief review of BPS Alice strings. In Sect. 3, we discuss the translational and $U(1)$ moduli separately. In Sect. 4 we derive the effective action of the zero modes, and finally we give a summary of our results and discussions in Sect. 5, in which we discuss a possible interaction of the $U(1)$ mode and the $U(1)$ gauge field in the bulk.

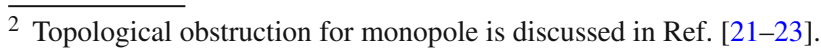

\section{BPS Alice strings}

In this section, we give a short review of BPS Alice strings; for details see Ref. [1]. We consider an $U(1) \times S O(3)$ gauge theory in this paper. However, we use $S U(2)$ notations for convenience. The corresponding action can be written as

$$
\begin{aligned}
I= & \int \mathrm{d}^{4} x\left[-\frac{1}{2} \operatorname{Tr} F_{\mu \nu} F^{\mu \nu}-\frac{1}{4} f_{\mu \nu} f^{\mu \nu}+\operatorname{Tr}\left|D_{\mu} \Phi\right|^{2}\right. \\
& \left.-\frac{\lambda_{g}}{4} \operatorname{Tr}\left[\Phi, \Phi^{\dagger}\right]^{2}-\frac{\lambda_{e}}{2}\left(\operatorname{Tr} \Phi \Phi^{\dagger}-2 \xi^{2}\right)^{2}\right],
\end{aligned}
$$

where $D_{\mu} \Phi=\partial_{\mu} \Phi-i e a_{\mu} \Phi-i g\left[A_{\mu}, \Phi\right]$, and $F_{\mu \nu}=$ $\partial_{\mu} A_{\nu}-\partial_{\nu} A_{\mu}-i g\left[A_{\mu}, A_{\nu}\right], f_{\mu \nu}=\partial_{\mu} a_{\nu}-\partial_{\nu} a_{\mu}$, with gauge couplings $g$ and $e$ for $S U(2)$ and $U(1)$ gauge fields, respectively. Here we have three charged complex fields in the triplet representation; $\Phi=\Phi^{a} \tau^{a}$ interacting with $S U$ (2) and $U(1)$ gauge fields $A_{\mu}$ and $a_{\mu}$, respectively. ${ }^{3}$ The $U(1)$ symmetry gives the stability to a fractional vortex and we can have an unbroken $\mathbb{Z}_{2}$ in the vacuum. We can choose the vacuum expectation value of the field $\Phi$ thus:

$\langle\Phi\rangle_{v}=2 \xi \tau^{1}$

with $\tau^{1}=\frac{1}{2} \sigma^{1}$. This breaks the gauge symmetry group $G=$ $U(1) \times S O(3) \simeq U(1) \times \frac{S U(2)}{\mathbb{Z}_{2}}$ as

$G=U(1) \times S O(3) \simeq U(1) \times \frac{S U(2)}{\mathbb{Z}_{2}}$

$\longrightarrow H=\mathbb{Z}_{2} \ltimes U(1)_{1} \simeq O(2)$,

where $\ltimes$ stands for a semi-direct product. Here in $U(1)_{1}$ the suffix 1 stands for rotation around $\tau^{1}$. The meaning of the semi-direct product can be understood as follows. Equation (2) says that any rotation around $\tau^{1}$ keeps $\langle\Phi\rangle_{v}$ invariant. The unbroken discrete group $\mathbb{Z}_{2}$ is defined as a simultaneous $\pi$ rotation around axes directed along any linear combination of $\tau^{3}$ and $\tau^{2}$ and in $U(1)$. This keeps $\langle\Phi\rangle_{v}$ invariant since the two $\pi$ rotations generate sign changes separately. The unbroken group elements can be written as

$H=\left\{\left(1, \mathrm{e}^{\frac{i \alpha}{2} \sigma^{1}}\right),\left(-1, i\left(c_{2} \sigma^{2}+c_{3} \sigma^{3}\right) \mathrm{e}^{i \frac{\alpha}{2} \sigma^{1}}\right)\right\}$,

where $c_{2}, c_{3}$ are arbitrary real constants normalized to the unity as $c_{2}^{2}+c_{3}^{2}=1$. The semi-direct product is arising here because the $\mathbb{Z}_{2}$ element changes the action of $U(1)_{1}$. The fundamental group for this symmetry breaking can be written as

$\pi_{1}\left(\frac{U(1) \times S O(3)}{O(2)}\right) \simeq \pi_{1}\left(\frac{S^{1} \times S^{2}}{\mathbb{Z}_{2}}\right) \simeq \mathbb{Z}$.

\footnotetext{
3 The cosmic strings in the same theory have also been discussed before in Ref. [28]. However, an explicit Bogomol'nyi completion and BPS vortex solutions were not discussed there, and the authors of this reference seem to be unaware of the fact that these are Alice strings even though we have the same theory.
} 
This non-trivial fundamental group supports the existence of stable strings. The $\mathbb{Z}_{2}$ element makes the generator of unbroken $U(1)_{1}$ globally undefined in the presence of a string: the generator of the unbroken $U(1)$ changes sign as it encircles the string once, and this property of the vortex in this system identifies it as an Alice string.

Here we are studying BPS vortices and the BPS completion can be performed if we consider the critical couplings $\lambda_{e}=e^{2}$ and $\lambda_{g}=g^{2}$. In this case, the theory can be embedded into a supersymmetric gauge theory in which Alice strings preserve a half of the supersymmetry [1], but we do not focus on such an aspect in this paper. The Bogomol'nyi completion of the tension, that is, the static energy per a unit length, is found to be

$$
\begin{aligned}
\mathcal{T}= & \int \mathrm{d}^{2} x\left[\operatorname{Tr}\left[F_{12} \pm \frac{g}{2}\left[\Phi, \Phi^{\dagger}\right]\right]^{2}+\operatorname{Tr}\left|\mathcal{D}_{ \pm} \Phi\right|^{2}\right. \\
& \left.+\frac{1}{2}\left[f_{12} \pm e\left(\operatorname{Tr} \Phi \Phi^{\dagger}-2 \xi^{2}\right)\right]^{2} \pm 2 e f_{12} \xi^{2}\right] \\
\geq & 2 e \xi^{2}\left|\int \mathrm{d}^{2} x f_{12}\right|,
\end{aligned}
$$

with $\mathcal{D}_{ \pm} \equiv \frac{D_{1} \pm i D_{2}}{2}$. The saturation of the inequality in the above equation, implying the minimum tension of vortex solution in the same topological sector, yields BPS equations given by

$$
\begin{aligned}
& f_{12} \pm e\left(\operatorname{Tr} \Phi \Phi^{\dagger}-2 \xi^{2}\right)=0 \\
& F_{12} \pm \frac{g}{2}\left[\Phi, \Phi^{\dagger}\right]=0 \\
& \mathcal{D}_{ \pm} \Phi=\left(\mathcal{D}_{ \pm} \Phi\right)^{\dagger}=0 .
\end{aligned}
$$

In order to solve these equations for a single vortex solution, we take the vortex ansatz of the scalar and gauge fields,

$$
\begin{aligned}
\Phi(r, \theta) & =\xi\left(\begin{array}{cc}
0 & f_{1}(r) \mathrm{e}^{i \theta} \\
f_{2}(r) & 0
\end{array}\right), \\
a_{i}(r, \theta) & =-\frac{1}{2 e} \frac{\epsilon_{i j x_{j}}}{r^{2}} a(r), \\
A_{i}(r, \varphi) & =-\frac{1}{4 g} \frac{\epsilon_{i j x_{j}}}{r^{2}} \sigma^{3} A(r),
\end{aligned}
$$

where $\{r, \theta\}$ are radial and angular coordinates of the two dimensional space, respectively. The equations for the profile functions $f_{1}(r), f_{2}(r), A(r)$ and $a(r)$, depending only on the radial coordinate, are the same as those of non-Abelian vortices [29-36], and they can be solved numerically with the boundary conditions

$$
\begin{array}{cc}
f_{1}(0)=f_{2}^{\prime}(0)=0, & f_{1}(\infty)=f_{2}(\infty)=1, \\
A(0)=a(0)=0, & A(\infty)=a(\infty)=1 .
\end{array}
$$

The numerical solution is displayed in Fig. 1.

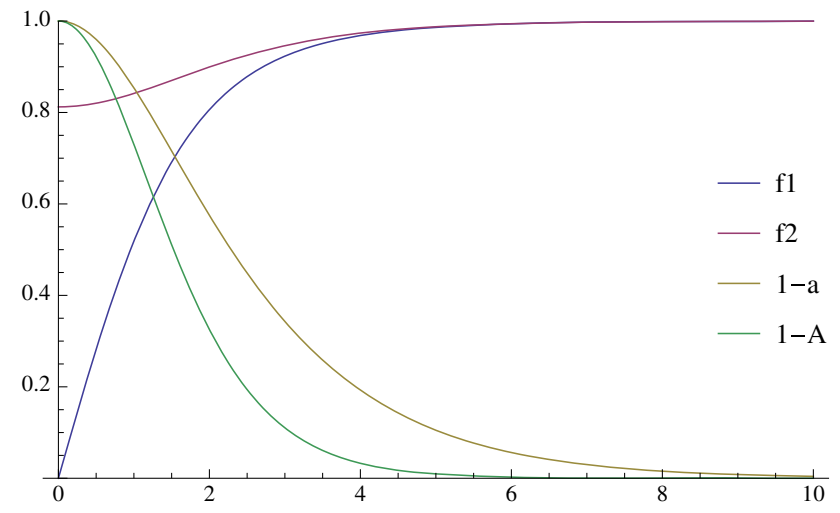

Fig. 1 The numerical solutions of the profile functions $f_{1}(r), f_{2}(r)$, $1-a(r)$ and $1-A(r)$ are displayed for a vortex configuration of winding number one as a function of $r$ (the distance from the vortex center) for $l=\frac{e}{g}=0.5[1]$

\section{Unbroken $U(1)$ symmetry}

The vacuum configuration in Eq. (2) shows that it is invariant under the adjoint action of the $U(1)$ subgroup generated by $\tau^{1}=\frac{1}{2} \sigma^{1}$. However, in the presence of a string, the scalar field configuration in Eq. (10) depends on space coordinates even at very large distances as

$$
\begin{aligned}
\Phi(R, \varphi) & \sim \xi \mathrm{e}^{i \frac{\theta}{2}}\left(\begin{array}{cc}
0 & \mathrm{e}^{i \frac{\theta}{2}} \\
\mathrm{e}^{-i \frac{\theta}{2}} & 0
\end{array}\right) \\
& =\Omega_{0}(\theta) \Omega_{3}(\theta) \Phi(R, 0) \Omega_{3}^{-1}(\theta),
\end{aligned}
$$

with $\Phi(R, \theta=0)=\xi \sigma^{1}$. The holonomy $\Omega$ can be defined by

$$
\Omega_{0}(\theta)=\mathrm{e}^{i e \int_{0}^{\theta} \mathbf{a} \cdot \mathbf{d l} \mathbf{l}}=\mathrm{e}^{i \frac{\theta}{2}}, \quad \Omega_{3}(\theta)=P \mathrm{e}^{i g \int_{0}^{\theta} \mathbf{A} \cdot \mathbf{d l}}=\mathrm{e}^{i \frac{\theta}{4} \sigma^{3}} .
$$

So the embedding of the unbroken $U$ (1) group becomes space dependent and the generator must be changed by the holonomies as it goes around the string as

$Q_{\theta}=\Omega_{3}(\theta) Q_{0} \Omega_{3}(\theta)^{-1}$.

After a full encirclement it is easy to find that $\Omega_{3}(2 \pi) \in \mathbb{Z}_{2}$ and

$Q_{2 \pi}=-Q_{0}=\mathrm{e}^{i 2 \pi \zeta} Q_{0}$,

where the 'obstruction' parameter $\zeta=\frac{1}{2}$. We shall see that $\mathbb{Z}_{2}$ affects the zero mode solution in the next section.

One comment is in order. A global version of the Alice string in our theory was already found in Ref. [37] (see Refs. $[38,39])$ in the context of Bose-Einstein condensation of ultra cold atomic gases. In the same context, a global monopole as a twisted Alice ring was also found [40]. By gauging $U(1) \times S O(3)$ symmetry, we obtained our theory which allows BPS Alice strings for the critical coupling [1]. 


\section{The moduli of a BPS Alice string}

Zero modes arise due to the breaking of any continuous unbroken bulk symmetry in the presence of a vortex solution. We can have continuously degenerate BPS solutions, which keeps the BPS equation and tension invariant. So we have a moduli space of solutions, and the motion on the moduli space generates zero modes. Here we would like to discuss translational and $U(1)$ zero modes separately.

\subsection{Translational moduli}

In this subsection, we discuss translational modes arising due to breaking of translational invariance, which are almost the same as other BPS vortices. The solution of the BPS equations can be shifted to any arbitrary point as the center of a vortex. So the zero modes can be found by expanding the BPS solutions around the origin which is taken as the center of our vortex. In a particular gauge, the zero mode solutions look like

$a_{i}^{T}(x)=-f_{m i} \delta X_{m}, \quad A_{i}^{T}(x)=-F_{m i}(x) \delta X_{m}$,

$\Phi^{T}(x)=-\mathcal{D}_{m} \Phi(x) \delta X_{m}$,

where $f_{m i}$ and $F_{m i}(x)$ are the field strengths computed from the BPS solutions and $\delta X_{m}$ is the displacement of the center of the vortex from the origin, where we place the vortex.

\section{$3.2 U(1)$ modulus}

As is well known zero modes arise when a soliton solution is not invariant under the action of a continuous unbroken symmetry group. In the case of the Alice string the unbroken $U(1)$ symmetry in the bulk is spontaneously broken in the core of the vortex. This can be observed easily when applying $U(1)$ transformation on the order parameter. On the $x$-axis (at $\theta=0$ ) the order parameter can be written as

$\Phi(r, 0)=\xi\left(\begin{array}{cc}0 & f_{1}(r) \\ f_{2}(r) & 0\end{array}\right)$.

Any small change due to the action of the $U(1)$ group elements $\mathrm{e}^{i \frac{\varphi}{2} \sigma^{1}}$ for small $\varphi$ is written as

$\delta \Phi(r, 0)=i \frac{\varphi}{2}\left[\sigma^{1}, \Phi(r, 0)\right]=i \frac{\varphi \xi}{2}\left(f_{2}(r)-f_{1}(r)\right) \sigma^{3}$.

At large distances from the vortex center, the order parameter is invariant under the $U(1)$ action because of $f_{1}(\infty)=$ $f_{2}(\infty)=1$. This is, however, not the case around the vortex core because of $f_{1}(r) \neq f_{2}(r)$ inside the vortex, according to the solution displayed in Fig. 1. The $U(1)$ transformation changes the magnetic flux as well at the vortex core, and so it generates a physically distinct degenerate solution.
Namely, if $\left\{\Phi, A_{i}\right\}$ minimizes the energy, then $\left\{\Phi(\varphi), A_{i}(\varphi)\right\}$ also does, where $\left\{\Phi(\varphi), A_{i}(\varphi)\right\}$ is defined by a global $U(1)$ transformation,

$\Phi(\varphi)=U_{\varphi} \Phi U_{\varphi}^{\dagger}, \quad A_{i}(\varphi)=U_{\varphi} A_{i} U_{\varphi}^{\dagger}, \quad U_{\varphi} \in U(1)$,

for a constant parameter $\varphi$. It is also true if $\varphi$ is a function of the $x, y$-coordinates, in which case we have to add an inhomogeneous term to the gauge field [17]. Since the $U(1)$ symmetry is broken inside the vortex we stick to a global transformation. This would generate an infinite set of solutions which are physically distinct. So $\varphi$ can be treated as a $U$ (1) modulus.

\section{The effective action of a BPS Alice string}

In the previous section we have discussed the existence of the zero modes by heuristic arguments. In this section, we construct the effective action of these zero modes by the moduli approximation $[41,42]$.

\subsection{The effective action of the translational moduli}

Let us start by shifting the center of the vortex to $(X(z, t)$, $Y(z, t))$; then the solution of the BPS equations becomes

$$
\begin{aligned}
& a_{i}\left(x_{m}-X_{m}(z, t)\right), \quad A_{i}\left(x_{m}-X_{m}(z, t)\right), \\
& \Phi\left(x_{m}-X_{m}(z, t)\right) .
\end{aligned}
$$

Here, we assume a slow variation of the vortex center with the moduli space approximation. This slow variation generates a non-zero current along the $t-z$ directions, and we have to introduce $t, z$ derivatives to solve the Gauss law. We may write the $\alpha=\{0,3\}$ derivative terms as

$$
f_{i \alpha}=V_{\alpha}^{j} \partial_{j} a_{i}, \quad F_{i \alpha}=V_{\alpha}^{j} \partial_{j} A_{i}, \quad \partial_{\alpha} \Phi=-V_{\alpha}^{j} \partial_{j} \Phi .
$$

Here the velocity is defined as $V_{\alpha}^{j}=\partial_{\alpha} X^{j}$. Let us choose a gauge so that the above expressions can be rewritten as

$f_{i \alpha}=V_{\alpha}^{j} f_{j i}, \quad F_{i \alpha}=V_{\alpha}^{j} F_{j i}, \quad \partial_{\alpha} \Phi=-V_{\alpha}^{j} \mathcal{D}_{j} \Phi$.

Here we are considering only small fluctuations, and we have neglected all higher order terms. Now using the first two terms, we may write

$\frac{1}{2} f_{i \alpha}^{2}=\frac{1}{4}\left(V_{\alpha}^{k}\right)^{2} f_{i j}^{2}, \quad \operatorname{Tr} F_{i \alpha}^{2}=\frac{1}{2}\left(V_{\alpha}^{k}\right)^{2} \operatorname{Tr} F_{i j}^{2}$.

By using the BPS equations, we may rewrite the above equations as

$$
\begin{aligned}
\frac{1}{2} f_{i \alpha}^{2} & =\frac{1}{2}\left(V_{\alpha}^{k}\right)^{2}\left[\frac{1}{4} f_{i j}^{2}+\frac{e^{2}}{2}\left(\operatorname{Tr} \Phi \Phi^{\dagger}-2 \xi^{2}\right)^{2}\right], \\
\operatorname{Tr} F_{i \alpha}^{2} & =\frac{1}{2}\left(V_{\alpha}^{k}\right)^{2}\left[\frac{1}{2} \operatorname{Tr} F_{i j}^{2}+\frac{g^{2}}{4} \operatorname{Tr}\left[\Phi, \Phi^{\dagger}\right]^{2}\right] .
\end{aligned}
$$


Now using rotational invariance and the BPS equation, $\mathcal{D}_{+} \Phi=0$, we may write

$$
\int d^{2} x\left|\partial_{\alpha} \Phi\right|^{2}=\frac{1}{2}\left(V_{\alpha}^{k}\right)^{2} \int d^{2} x \operatorname{Tr}\left|\mathcal{D}_{i} \Phi\right|^{2} .
$$

We now use Eqs. (26), (27) and (28), and following Eq. (6) and the solutions of the BPS equations the effective action is written as

$$
\int d^{2} x\left[\frac{1}{2} f_{i \alpha}^{2}+\operatorname{Tr} F_{i \alpha}^{2}+\operatorname{Tr}\left|\mathcal{D}_{\alpha} \Phi\right|^{2}\right]=\pi \xi^{2}\left(\partial_{\alpha} X^{k}\right)^{2} .
$$

\subsection{The effective action of the $U(1)$ modulus}

To understand the behavior of the $U(1)$ modulus we need to excite the modes along the $z$-axis to write down the effective action. We set the fluctuation of the $U(1)$ modulus of the scalar field as

$$
\begin{gathered}
\Phi(\varphi(t, z), r, \theta=0)=U_{\varphi}(t, z) \Phi(r) U_{\varphi}^{\dagger}(t, z), \\
U_{\varphi}(t, z)=\mathrm{e}^{i \varphi(t, z) \tau^{1}},
\end{gathered}
$$

where $\varphi(t, z)$ is taken as a slowly varying function on the $t-z$ plane. Since the generator of unbroken group is not well defined globally, as described in Ref. [1], we define the $\theta$ dependence of the scalar as

$$
\begin{aligned}
& \Phi(\varphi(t, z), r, \theta) \\
& =\mathrm{e}^{i \frac{\theta}{2}} U_{\varphi}(\theta, t, z) \Phi(\varphi(t, z), r, \theta=0) U_{\varphi}^{\dagger}(\theta, t, z), \\
& \quad U_{\varphi}(\theta, t, z)=\mathrm{e}^{\frac{i \theta}{4} U_{\varphi} \sigma^{3} U_{\varphi}^{\dagger}} .
\end{aligned}
$$

This can be rewritten as

$$
\begin{aligned}
\Phi(\varphi(t, z), r, \theta) & =\mathrm{e}^{i \frac{\varphi}{2} \sigma^{1}}\left(\begin{array}{cc}
0 & f_{1}(r) \mathrm{e}^{i \theta} \\
f_{2}(r) & 0
\end{array}\right) \mathrm{e}^{-i \frac{\varphi}{2} \sigma^{1}} \\
& =U_{\varphi}(t, z) \Phi(r, \theta) U_{\varphi}^{\dagger}(t, z) .
\end{aligned}
$$

Similarly we can introduce the gauge field fluctuation corresponding to the $U(1)$ modulus by

$$
A_{i}(\varphi(t, z), x, y)=-\frac{1}{4 g} \frac{\epsilon_{i j x_{j}}}{r^{2}} A(r) U_{\varphi}(t, z) \sigma^{3} U_{\varphi}^{\dagger}(t, z) .
$$

The $(t, z)$ dependence of the fluctuations may keep the BPS equations unchanged, but it would generate $J_{0}$ and $J_{3}$ in the equations of motion of the gauge field. This violates the Gauss law and the Biot-Savart law, and we need to introduce $A_{0}$ and $A_{3}$ to solve these equations. Since $F_{\alpha \beta}=0$, we may write the effective action as

$$
\begin{aligned}
I_{\text {eff }}= & \int \mathrm{d} t \mathrm{~d} z\left[\int \mathrm { d } ^ { 2 } x \left\{-\frac{1}{2} f_{i \alpha} f^{i \alpha}-\operatorname{Tr} F_{i \alpha} F^{i \alpha}\right.\right. \\
& \left.\left.+\operatorname{Tr}\left|D_{\alpha} \Phi(\varphi)\right|^{2}\right\}\right], \quad \alpha=\{0,3\},
\end{aligned}
$$

where $F_{i \alpha}=\partial_{i} A_{\alpha}-D_{\alpha} A_{i}(\varphi), D_{\alpha}=\partial_{\alpha}-i g\left[A_{\alpha}, \cdot\right]$, $D_{\alpha} \Phi(\varphi)=\partial_{\alpha} \Phi(\varphi)-i e a_{\alpha} \Phi(\varphi)-i g\left[A_{\alpha}, \Phi(\varphi)\right]$. Equation (34) is a part of the full action, and the rest vanishes for the BPS solutions.

Now by variations of $a_{\alpha}$ and $A_{\alpha}$, we may write the equations of motion as

$$
\begin{aligned}
\partial_{i} f_{i \alpha} & =i e \operatorname{Tr}\left[\Phi^{\dagger} D_{\alpha} \Phi-\Phi\left(D_{\alpha} \Phi\right)^{\dagger}\right], \\
D_{i} F_{i \alpha} & =-i \frac{g}{2}\left\{\left[\Phi^{\dagger}, D_{\alpha} \Phi\right]+\left[\Phi,\left(D_{\alpha} \Phi\right)^{\dagger}\right]\right\} .
\end{aligned}
$$

To solve these equations of motion we introduce an ansatz of the generated components $A_{\alpha}$ of the gauge field as

$$
\begin{aligned}
A_{\alpha}(t, z, r, \theta) & =\frac{1}{g}\left[\left(1-\Psi_{1}(r, \theta)\right) \tau^{1}+\Psi_{2}(r, \theta) T^{2}\right] \partial_{\alpha} \varphi(t, z), \\
a_{\alpha} & =0, \quad T^{2}=U_{\varphi}(t, z) \tau^{2} U_{\varphi}^{\dagger}(t, z), \quad \tau^{i}=\frac{1}{2} \sigma^{i} .
\end{aligned}
$$

Here $\Psi_{i}(r, \theta)$ are real and imaginary components of an arbitrary complex function on the $x-y$ plane and these can be found by solving the equations of motion. To find the profile functions $\Psi_{i}(r, \theta)$, we may insert the ansatz into the equations of motion in Eq. (35) and solve the equations for $\Psi_{i}(r, \theta)$. Likewise we may insert the ansatz into the effective action in Eq. (34) and find the equations for $\Psi_{i}$ after a variation with respect to $\Psi_{i}$. Since the two cases give the same equations of $\Psi_{i}$, we use the second calculation here. It is straightforward to show that the above ansatz in Eq. (36) solves the equations of motion in Eq. (35). Now we may use the zero mode ansatz in Eq. (36) and the vortex solution ansatz in Eq. (32) and (33) to express $F_{i \alpha}$ and $D_{\alpha} \Phi$ in terms of $\Psi_{i}$ and $\varphi$ as

$$
\begin{aligned}
F_{i \alpha} & =\frac{\partial_{\alpha} \varphi}{g}\left[-\left(\partial_{i} \Psi_{1}+\zeta b_{i} \Psi_{2}\right) \tau^{1}+\left(\partial_{i} \Psi_{2}-\zeta b_{i} \Psi_{1}\right) T^{2}\right], \\
D_{\alpha} \Phi & =-\mathrm{e}^{i \frac{\theta}{2}} g \xi\left[\Psi_{1} \Phi_{2}+\Psi_{2} \Phi_{1}\right] \partial_{\alpha} \varphi T^{3},
\end{aligned}
$$

where the "obstruction" parameter is $\zeta=\frac{1}{2}, b_{i}=$ $-\frac{\epsilon_{i j} x_{j}}{r^{2}} A(r)$ and $T^{3}=U_{\varphi}(t, z) \tau^{3} U_{\varphi}^{\dagger}(t, z)$. Here $\Phi_{1}$ and $\Phi_{2}$ have been defined by

$$
\begin{aligned}
& \Phi_{1}(r, \theta)=\left(f_{1}(r) \mathrm{e}^{i \frac{\theta}{2}}+f_{2}(r) \mathrm{e}^{-i \frac{\theta}{2}}\right), \\
& \Phi_{2}(r, \theta)=i\left(f_{1}(r) \mathrm{e}^{i \frac{\theta}{2}}-f_{2}(r) \mathrm{e}^{-i \frac{\theta}{2}}\right) .
\end{aligned}
$$

After inserting the expressions in Eq. (37) into the effective action in Eq. (34) we find

$$
\begin{aligned}
\mathcal{L}_{\text {eff }}= & \operatorname{Tr} F_{i \alpha} F_{i}^{\alpha}+\operatorname{Tr}\left(D_{\alpha} \Phi\right)^{\dagger} D^{\alpha} \Phi \\
= & {\left[\left(\partial_{i} \Psi_{1}+\zeta b_{i} \Psi_{2}\right)^{2}+\left(\partial_{i} \Psi_{2}-\zeta b_{i} \Psi_{1}\right)^{2}\right.} \\
& \left.+g^{2} \xi^{2}\left|\Psi_{1} \Phi_{2}+\Psi_{2} \Phi_{1}\right|^{2}\right] \frac{\partial_{\alpha} \varphi \partial^{\alpha} \varphi}{2 g^{2}} .
\end{aligned}
$$

It can be observed here that the derivative terms of the above effective action is invariant under an $S O(2)$ transformation 
generated by $T=\left(\begin{array}{cc}0 & 1 \\ -1 & 0\end{array}\right)$. This leads us to define a complex function

$\Psi(r, \theta)=\Psi_{1}(r, \theta)+i \Psi_{2}(r, \theta)$.

By using this complex function, the above effective action in Eq. (39) can be rewritten in a simpler form as

$$
\begin{aligned}
\mathcal{I}_{\text {eff }} & =\int \mathrm{d}^{2} x\left[\left|D_{i} \Psi\right|^{2}+\Delta_{g}^{2}\left|\Psi^{*} q_{1}-\Psi q_{2}\right|^{2}\right] \int \mathrm{d} t \mathrm{~d} z\left\{\frac{\partial_{\alpha} \varphi \partial^{\alpha} \varphi}{2 g^{2}}\right\}, \\
& =I_{\Psi} \int d t d z\left\{\frac{\partial_{\alpha} \varphi \partial^{\alpha} \varphi}{2 g^{2}}\right\}, \quad \Delta_{g}^{2}=\xi^{2} g^{2},
\end{aligned}
$$

where $D_{i} \Psi=\left(\partial_{i}-i \zeta b_{i}\right) \Psi$ and

$I_{\Psi}=\int \mathrm{d}^{2} x\left[\left|D_{i} \Psi\right|^{2}+\Delta_{g}^{2}\left|\Psi^{*} q_{1}-\Psi q_{2}\right|^{2}\right]$,

$q_{1}=f_{1} \mathrm{e}^{i \frac{1}{2} \theta}, q_{2}=f_{2} \mathrm{e}^{-i \frac{1}{2} \theta}$.

We should notice here that $\Psi$ behaves as if a scalar field in $1+$ 1 dimensions in the background of the vortex Abelian gauge field $b_{i}$ with a charge $\zeta=\frac{1}{2}$. The important thing to note is that the $(x, y)$ and $(t, z)$ dependent parts are completely separated. So we may integrate over the $x-y$ plane and get the $1+1$ dimensional vortex effective action. To evaluate the front factor we extremize $I_{\Psi}$ by varying $\Psi$ and find the equation for $\Psi$,

$D_{i}^{2} \Psi-\Delta_{g}^{2}\left[\left(f_{1}^{2}+f_{2}^{2}\right) \Psi-2 f_{1} f_{2} \mathrm{e}^{i \theta} \Psi^{*}\right]=0$.

We may write the equation in polar coordinates $(r, \theta)$ as

$$
\begin{aligned}
& \frac{1}{\rho} \frac{\partial}{\partial \rho}\left(\rho \frac{\partial \Psi}{\partial \rho}\right)+\left(\frac{\partial_{\theta}-i \zeta A(\rho)}{\rho}\right)^{2} \Psi \\
& -\left[\left(f_{1}^{2}+f_{2}^{2}\right) \Psi-2 f_{1} f_{2} \mathrm{e}^{i \theta} \Psi^{*}\right]=0,
\end{aligned}
$$

where we have made the above equation dimensionless by defining a rescaled length, $\rho=\Delta_{g} r$.

In order to find solutions of this equation, let us decompose the complex profile function $\Psi$ into partial wave modes by $\Psi(\rho, \theta)=\sum_{m \geq 0}\left[a_{m}(\rho) \mathrm{e}^{i m \theta}+b_{m}(\rho) \mathrm{e}^{-i m \theta}\right], \quad a_{0}(\rho)=0$,

for $m=0, \Psi$ would only be described by $b_{0}(\rho)$. After inserting the expansion of Eq. (45) into Eq. (44) we find the coupled equations of the infinite tower of pairs $\left\{a_{m}(\rho), b_{m}(\rho)\right\}$ as

$$
\begin{gathered}
\frac{1}{\rho} \frac{\partial}{\partial \rho}\left(\rho \frac{\partial a_{m}}{\partial \rho}\right)-\left(\frac{m-\zeta A(\rho)}{\rho}\right)^{2} a_{m} \\
-\left[\left(f_{1}^{2}+f_{2}^{2}\right) a_{m}-2 f_{1} f_{2} b_{m-1}\right]=0, \\
\frac{1}{\rho} \frac{\partial}{\partial \rho}\left(\rho \frac{\partial b_{m}}{\partial \rho}\right)-\left(\frac{m+\zeta A(\rho)}{\rho}\right)^{2} b_{m} \\
-\left[\left(f_{1}^{2}+f_{2}^{2}\right) b_{m}-2 f_{1} f_{2} a_{m+1}\right]=0 .
\end{gathered}
$$

From now on, we would like to show that the following expressions give a solution to Eqs. (46) and (47):

$a_{m}(\rho)=\rho^{m-\zeta} f(\rho)^{\zeta}, \quad$ for $m>0$,

$b_{m}(\rho)=\rho^{m+\zeta} f(\rho)^{-\zeta}, \quad$ for $m \geq 0$.

Inserting these solutions into the last terms of Eqs. (46) and (47) yields (by using $1-\zeta=\zeta$ )

$$
\begin{aligned}
& {\left[\left(f_{1}^{2}+f_{2}^{2}\right) a_{m}-2 f_{1} f_{2} b_{m-1}\right]=\left[f_{1}^{2}-f_{2}^{2}\right] a_{m}(\rho),} \\
& {\left[\left(f_{1}^{2}+f_{2}^{2}\right) b_{m}-2 f_{1} f_{2} a_{m+1}\right]=-\left[f_{1}^{2}-f_{2}^{2}\right] b_{m}(\rho),}
\end{aligned}
$$

where we have used the identity $f_{1}(\rho) f(\rho)^{-\zeta}=f_{2}(\rho)$ $f(\rho)^{1-\zeta}=f_{2}(\rho) f(\rho)^{\zeta}$. Then we have

$$
\begin{aligned}
& \frac{1}{\rho} \frac{\partial}{\partial \rho}\left(\rho \frac{\partial a_{m}}{\partial \rho}\right)-\left(\frac{m-\zeta A(\rho)}{\rho}\right)^{2} a_{m} \\
& -\left[f_{1}^{2}-f_{2}^{2}\right] a_{m}(\rho)=0, \\
& \frac{1}{\rho} \frac{\partial}{\partial \rho}\left(\rho \frac{\partial b_{m}}{\partial \rho}\right)-\left(\frac{m+\zeta A(\rho)}{\rho}\right)^{2} b_{m} \\
& \quad+\left[f_{1}^{2}-f_{2}^{2}\right] b_{m}(\rho)=0 .
\end{aligned}
$$

In order to show that these equations hold, let us use the BPS equations. Following Ref. [1] we may express the BPS equations for the profile functions $f_{1}(\rho), f_{2}(\rho)$ and $h_{A}(\rho)=$ $1-A(\rho)$ as

$$
\begin{gathered}
\frac{1}{\rho} \frac{\partial}{\partial \rho}\left(h_{A}(\rho)\right)-2\left[f_{1}(\rho)^{2}-f_{2}(\rho)^{2}\right]=0, \\
h_{A}(\rho)=\rho \frac{\partial}{\partial \rho} \log f(\rho) .
\end{gathered}
$$

Here $f(\rho)$ is defined as $f(\rho)=\frac{f_{1}(\rho)}{f_{2}(\rho)}$. By defining a function $f_{m}(\rho)=\rho^{m-\xi} f(\rho)^{\xi}$, this equation can be rewritten as

$\rho \frac{\partial}{\partial \rho} f_{m}(\rho)=(m-\xi A(\rho)) f_{m}(\rho)$,

where $\xi$ is any real number. By applying $\left(\frac{1}{\rho} \partial_{\rho}\right)$ from the left and by using Eq. (51) again, this equation can be rewritten as

$$
\begin{aligned}
& \frac{1}{\rho} \frac{\partial}{\partial \rho}\left(\rho \frac{\partial}{\partial \rho} f_{m}(\rho)\right)=\left(\frac{m-\xi A(\rho)}{\rho}\right)^{2} f_{m}(\rho) \\
& \quad+2 \xi\left[f_{1}(\rho)^{2}-f_{2}(\rho)^{2}\right] f_{m}(\rho) .
\end{aligned}
$$

So for $\xi= \pm \zeta$, this reduces to

$$
\begin{gathered}
\frac{1}{\rho} \frac{\partial}{\partial \rho}\left(\rho \frac{\partial}{\partial \rho} f_{m}(\rho)\right)-\left(\frac{m \mp \zeta A(\rho)}{\rho}\right)^{2} f_{m}(\rho) \\
\mp 2 \zeta\left[f_{1}(\rho)^{2}-f_{2}(\rho)^{2}\right] f_{m}(\rho)=0 .
\end{gathered}
$$


This equation is the same as Eq. (50) if we identify $f_{m}$ with $a_{m}$ or $b_{m}$ for $\zeta=\frac{1}{2}$. We thus have found that an exact solution to Eq. (50) is given by Eq. (48).

Let us discuss the asymptotic behaviors at large and short distances. At large distances the solutions diverge as

$a_{m}(\rho) \longrightarrow \rho^{m-\zeta}, \quad$ for $m>0$,

$b_{m}(\rho) \longrightarrow \rho^{m+\zeta}, \quad$ for $m \geq 0$,

since $f(\rho) \longrightarrow 1$ as $\rho \rightarrow \infty$. This can also be understood by analyzing the asymptotic behavior of Eq. (46). Since at large distances $f_{1}(\rho)=f_{2}(\rho)$, Eq. (46) has the solution $b_{m}(\rho)=a_{m+1}(\rho) \simeq \rho^{m+\zeta}$. This behavior at large distance is well known in the literature [17].

On the other hand, the short distance behavior was not known before. To describe the asymptotic behavior of the solution one should notice that at the center of the vortex the solution vanishes as $\sim \rho^{m}$ except for $b_{0}$. The $\rho$ dependence cancels for $b_{0}$ in the limit $\rho \longrightarrow 0$ since $f_{1}(\rho) \sim \rho$ as $\rho \longrightarrow 0$ and we find

$b_{0} \longrightarrow f_{2}(0)^{\frac{1}{2}}, \quad$ as $\rho \longrightarrow 0$,

where $f_{2}(0) \neq 0$ can be observed from the solution plot in Fig. 1.

Finally, let us analyze the energy by using the solution we found. The energy of the fluctuation mode is determined with the front factor $I_{\Psi}$ defined in Eq. (41) as

$\mathcal{E}=I_{\psi}\left\{\frac{\left(\partial_{\alpha} \varphi\right)^{2}}{2 g^{2}}\right\}$

To derive the value of $I_{\Psi}$ we have to use the equations of motion of $\Psi$ written in Eq. (43). We multiply $\Psi^{*}$ with Eq. (43) and add this with its complex conjugate equation to yield

$\Psi^{*} D_{i}^{2} \Psi+\left(D_{i}^{2} \Psi\right)^{*} \Psi=2 \Delta_{g}^{2}\left|\Psi^{*} q_{1}-\Psi q_{2}\right|^{2}$.

This relation would help us to compute $I_{\Psi}$ exactly as

$$
\begin{aligned}
I_{\Psi} & =\int \mathrm{d}^{2} x\left[\left|D_{i} \Psi\right|^{2}+\Delta_{g}^{2}\left|\Psi^{*} q_{1}-\Psi q_{2}\right|^{2}\right] \\
& =\frac{1}{2} \int \mathrm{d}^{2} x \nabla^{2}\left(\Psi^{*} \Psi\right) .
\end{aligned}
$$

If we consider the system within a large loop of dimensionless radius $R$, the above integral gives

$$
I_{\Psi}=\pi R+\pi \sum_{m>0}\left[(2 m-1) R^{2 m-1}+(2 m+1) R^{2 m+1}\right] .
$$

So the energy of the zero mode can be expressed as

$$
\begin{aligned}
\mathcal{E}= & \frac{2 \pi R}{4 g^{2}}\left[1+\sum_{m>0}\left\{(2 m-1) R^{2(m-1)}\right.\right. \\
& \left.\left.+(2 m+1) R^{2 m}\right\}\right]\left(\partial_{\alpha} \varphi\right)^{2} .
\end{aligned}
$$

For a zero mode of wavelength $\lambda$ in the $z$-direction the energy $(m=0)$ behaves as

$\mathcal{E}_{\lambda}=\frac{2 \pi R}{4 g^{2} \lambda^{2}}$.

So for fixed radius $R$ energy of very long wavelength the zero mode fluctuations vanish.

\section{Summary and discussion}

In this paper, we have studied in detail the zero modes of a single BPS Alice string. We have explicitly worked things out in a $U(1) \times S O(3)$ gauge theory with three complex scalar fields in the triplet representation. In this system, the $U(1) \times S O(3)$ symmetry is spontaneously broken to $\mathbb{Z}_{2} \ltimes U(1) \simeq O(2)$. This symmetry breaking can create Alice strings, since the fundamental group $\pi_{1}(G / H)=\mathbb{Z}$; this vortex has the Alice property, since the generator of the unbroken $U(1)$ symmetry changes sign as it encircles the string once, due to the $\mathbb{Z}_{2}$ factor of a semi-direct product in the unbroken symmetry. We constructed this vortex after the BPS completion in the previous paper. In the present paper we have discussed the $U(1)$ zero mode as well as the translational modes of a single BPS Alice string. The translational modes were found to be similar to other conventional vortices. The $U(1)$ mode is generated due to the spontaneous breaking of the unbroken $U$ (1) bulk symmetry inside the vortex core. By promoting the moduli parameters as the fields in the $(t, z)$ plane we introduced gauge fields $A_{\alpha}=\left\{A_{0}, A_{3}\right\}$ in the system to solve the Gauss law. The profile functions of these gauge fields are defined by a complex scalar. We have expanded the profile function in partial wave modes and found that the equations are exactly solvable in terms of the vortex profile functions $f_{1}(r)$ and $f_{2}(r)$ and this solution was not known so far for Alice strings. The partial wave modes have been found to be divergent in powers of the radial coordinate. After inserting the zero mode profile functions into the effective action, we have written down the effective energy, which is also found to be divergent. The minimum mode is divergent linearly as expected from the derivation in Ref. [16].

The reason for the divergent energy may be the fact that in our calculation we have not taken into account the massless bulk gauge field fluctuation effect. In principle, to write down an effective action by using the renormalization group, one should not integrate it out, since it is massless. In this situ- 
ation, by following Ref. [4], one may write down a $2 \mathrm{~d}-4 \mathrm{~d}$ action as (see Ref. [19]),

$\mathcal{I}_{2 d-4 d}=-\int \mathrm{d}^{4} x \frac{1}{4} F_{\mu \nu}^{2}+M_{r}^{2} \int \mathrm{d} t \mathrm{~d} z\left(\partial_{\alpha} \varphi+A_{\alpha}\right)^{2}$,

where $M_{r}$ is the regularized mass and the field strength can be defined with a branch cut as

$F_{\mu \nu}=\partial_{\mu} A_{\nu}-\partial_{\nu} A_{\mu}-2 \pi \zeta \Sigma_{\mu \nu}$.

Here, $\Sigma_{\mu \nu}$ is a branch cut orthogonal to the string world-sheet whose direction is determined by a singular gauge and can be changed by a singular gauge transformation. The electric charge changes its sign when it passes across the cut. The coefficient $2 \pi \zeta$ is an Aharonov-Bohm (AB) phase and in our case $\zeta=\frac{1}{2}$. Then the $\mathrm{AB}$ scattering of photons off an Alice string and $\mathrm{AB}$ scattering of two Alice strings should enable one to study it. In particular for the latter case, it can be dealt with within the moduli approximation due to the BPS nature, which was impossible for the conventional Alice strings.

In this paper, we have discussed bosonic zero modes in a bosonic theory. There appear fermion zero modes in the string core, once we couple the theory to fermions, such as supersymmetric extensions. Fermion zero modes will give rise to a non-Abelian statistics for both Majorana [43-46] and Dirac fermions $[47,48]$. This will be a novelty, since there are two origins of non-Abelian statistics: boson and fermion zero modes.

In this paper we have discussed zero mode excitations of a single Alice string and have found that the $U(1)$ zero mode is non-normalizable. It would be more interesting to study multi-string configurations because for an even number of strings there will be no obstruction globally. So one may expect to have a normalizable $U(1)$ mode for an even number of strings, and the system may possess a finite electric field, which is expected as regards the explanation of the existence of a delocalized Cheshire charge [17]. Since our Alice strings are BPS, we can consider a stable multi-vortex system. A similar zero mode whose wave functions are spread between solitons is known for BPS semi-local vortices [49]. It is also important to prove the index theorem for these vortices. It would be different from that of conventional vortices in the Abelian Higgs model, since there is a massless field present in the bulk. So the vanishing theorem does not work in this case. We shall address these issues in the future.

Acknowledgements This work is supported by the Ministry of Education, Culture, Sports, Science (MEXT)-Supported Program for the Strategic Research Foundation at Private Universities "Topological Science" (Grant No. S1511006). C. C. acknowledges support as an International Research Fellow of the Japan Society for the Promotion of Science (JSPS)(Grant No: 16F16322). The work of M. N. is supported in part by JSPS Grant-in-Aid for Scientific Research (KAKENHI Grant No. 16H03984), and by a Grant-in-Aid for Scientific Research on
Innovative Areas "Topological Materials Science" (KAKENHI Grant No. 15H05855) from the Ministry of Education, Culture, Sports, Science (MEXT) of Japan.

Open Access This article is distributed under the terms of the Creative Commons Attribution 4.0 International License (http://creativecomm ons.org/licenses/by/4.0/), which permits unrestricted use, distribution, and reproduction in any medium, provided you give appropriate credit to the original author(s) and the source, provide a link to the Creative Commons license, and indicate if changes were made.

Funded by $\mathrm{SCOAP}^{3}$.

\section{References}

1. C. Chatterjee, M. Nitta, BPS Alice strings. JHEP 1709, 046 (2017). arXiv:1703.08971 [hep-th]

2. T.W.B. Kibble, Topology of cosmic domains and strings. J. Phys. A 9, 1387 (1976)

3. M.B. Hindmarsh, T.W.B. Kibble, Cosmic strings. Rept. Prog. Phys. 58, 477 (1995). arXiv:hep-ph/9411342

4. E. Witten, Superconducting strings. Nucl. Phys. B 249, 557 (1985)

5. A.S. Schwarz, Field theories with no local conservation of the electric charge. Nucl. Phys. B 208, 141 (1982)

6. J.E. Kiskis, Disconnected gauge groups and the global violation of charge conservation. Phys. Rev. D 17, 3196 (1978)

7. J. Preskill, L.M. Krauss, Local discrete symmetry and quantum mechanical hair. Nucl. Phys. B 341, 50 (1990)

8. M. Bucher, A. Goldhaber, $\mathrm{SO}(10)$ cosmic strings and $\mathrm{SU}(3)$ color cheshire charge. Phys. Rev. D 49, 4167 (1994). arXiv:hep-ph/9310262

9. M. Bucher, H.K. Lo, J. Preskill, Topological approach to Alice electrodynamics. Nucl. Phys. B 386, 3 (1992). arXiv:hep-th/9112039

10. R. Shankar, More SO(3) monopoles. Phys. Rev. D 14, 1107 (1976)

11. F.A. Bais, J. Striet, On a core instability of 't Hooft-Polyakov monopoles. Phys. Lett. B 540, 319 (2002). arXiv:hep-th/0205152

12. J. Striet, F.A. Bais, More on core instabilities of magnetic monopoles. JHEP 0306, 022 (2003). arXiv:hep-th/0304189

13. K.M. Benson, T. Imbo, Topologically Alice strings and monopoles. Phys. Rev. D 70, 025005 (2004). arXiv:hep-th/0407001

14. M.G. Alford, F. Wilczek, Aharonov-Bohm interaction of cosmic strings with matter. Phys. Rev. Lett. 62, 1071 (1989)

15. H.K. Lo, J. Preskill, Non-Abelian vortices and non-Abelian statistics. Phys. Rev. D 48, 4821 (1993). arXiv:hep-th/9306006

16. M.G. Alford, K. Benson, S.R. Coleman, J. March-Russell, F. Wilczek, The interactions and excitations of nonabelian vortices. Phys. Rev. Lett. 64, 1632 (1990). (Erratum-ibid. 65 (1990) 668)

17. M.G. Alford, K. Benson, S.R. Coleman, J. March-Russell, F. Wilczek, Zeromodes of non-abelian vortices. Nucl. Phys. B 349, $414(1991)$

18. M.G. Alford, K.M. Lee, J. March-Russell, J. Preskill, Quantum field theory of non-Abelian strings and vortices. Nucl. Phys. B 384, 251 (1992)

19. S. Bolognesi, C. Chatterjee, J. Evslin, K. Konishi, K. Ohashi, L. Seveso, Geometry and dynamics of a coupled 4D-2D quantum field theory. JHEP 1601, 075 (2016). arXiv:1509.04061 [hep-th]

20. L.M. Krauss, F. Wilczek, Discrete gauge symmetry in continuum theories. Phys. Rev. Lett. 62, 1221 (1989)

21. P.C. Nelson, A. Manohar, Global color is not always defined. Phys. Rev. Lett. 50, 943 (1983)

22. A. Abouelsaood, Are there chromodyons? Nucl. Phys. B 226, 309 (1983)

23. A.P. Balachandran, G. Marmo, N. Mukunda, J.S. Nilsson, E.C.G. Sudarshan, F. Zaccaria, Monopole topology and the problem of color. Phys. Rev. Lett. 50, 1553 (1983) 
24. E. B. Bogomolny, Stability of classical solutions. Sov. J. Nucl. Phys. 24, 449 (1976). [Yad. Fiz. 24, 861 (1976)]

25. M.K. Prasad, C.M. Sommerfield, An exact classical solution for the 't Hooft Monopole and the Julia-Zee Dyon. Phys. Rev. Lett. 35, 760 (1975)

26. A. A. Abrikosov, On the magnetic properties of superconductors of the second group. Sov. Phys. JETP 5, 1174 (1957). [Zh. Eksp. Teor. Fiz. 32, 1442 (1957)]

27. H.B. Nielsen, P. Olesen, Vortex line models for dual strings. Nucl. Phys. B 61, 45 (1973)

28. S.C. Davis, A.C. Davis, M. Trodden, Cosmic strings, zero modes and SUSY breaking in non-Abelian $\mathrm{N}=1$ gauge theories. Phys. Rev. D 57, 5184 (1998). arXiv:hep-ph/9711313

29. A. Hanany, D. Tong, Vortices, instantons and branes. JHEP $\mathbf{0 3 0 7}$ 037 (2003). arXiv:hep-th/0306150

30. R. Auzzi, S. Bolognesi, J. Evslin, K. Konishi, A. Yung, NonAbelian superconductors: vortices and confinement in N=2 SQCD. Nucl. Phys. B 673, 187 (2003). arXiv:hep-th/0307287

31. M. Eto, Y. Isozumi, M. Nitta, K. Ohashi, N. Sakai, Moduli space of non-Abelian vortices. Phys. Rev. Lett. 96, 161601 (2006). arXiv:hep-th/0511088

32. M. Eto, K. Konishi, G. Marmorini, M. Nitta, K. Ohashi, W. Vinci, N. Yokoi, Non-Abelian vortices of higher winding numbers. Phys. Rev. D 74, 065021 (2006). arXiv:hep-th/0607070

33. M. Eto, K. Hashimoto, G. Marmorini, M. Nitta, K. Ohashi, W. Vinci, Universal reconnection of Non-Abelian cosmic strings. Phys. Rev. Lett. 98, 091602 (2007). arXiv:hep-th/0609214

34. M. Eto, Y. Isozumi, M. Nitta, K. Ohashi, N. Sakai, Solitons in the higgs phase: the moduli matrix approach. J. Phys. A 39, R315 (2006). arXiv:hep-th/0602170

35. D. Tong, Quantum vortex strings: a review. Annals Phys. 324, 30 (2009). arXiv:0809.5060 [hep-th]

36. M. Shifman, A. Yung, Supersymmetric solitons and how they help us understand non-Abelian gauge theories. Rev. Mod. Phys. 79, 1139 (2007). arXiv:hep-th/0703267

37. U. Leonhardt, G .E. Volovik, How to create Alice string (half quantum vortex) in a vector Bose-Einstein condensate. Pisma Zh. Eksp. Teor. Fiz. 72, 66 (2000). [JETP Lett. 72, 46 (2000)]. arXiv:cond-mat/0003428
38. S. Kobayashi, M. Kobayashi, Y. Kawaguchi, M. Nitta, M. Ueda, Abe homotopy classification of topological excitations under the topological influence of vortices. Nucl. Phys. B 856, 577 (2012). arXiv:1110.1478 [math-ph]

39. Y. Kawaguchi, M. Ueda, Spinor Bose-Einstein condensates. Phys. Rept. 520, 253 (2012). arXiv:1001.2072 [cond-mat.quant-gas]

40. J. Ruostekoski, J.R. Anglin, Monopole core instability and Alice rings in spinor Bose-Einstein condensates. Phys. Rev. Lett. 91, 190402 (2003). [Erratum: [Phys. Rev. Lett. 97, 069902 (2006)]. arXiv:cond-mat/0307651

41. N.S. Manton, A remark on the scattering of BPS monopoles. Phys. Lett. 110B, 54 (1982)

42. M. Eto, Y. Isozumi, M. Nitta, K. Ohashi, N. Sakai, Manifestly supersymmetric effective Lagrangians on BPS solitons. Phys. Rev. D 73, 125008 (2006). arXiv:hep-th/0602289

43. D. A. Ivanov, Non-abelian statistics of half-quantum vortices in p-wave superconductors. Phys. Rev. Lett. 86(2), 268 (2001). arXiv:cond-mat/0005069 [cond-mat.supr-con]

44. S. Yasui, K. Itakura and M. Nitta, "Majorana meets Coxeter: NonAbelian Majorana Fermions and Non-Abelian Statistics," Phys. Rev. B 83, 134518 (2011). arXiv:1010.3331 [cond-mat.mes-hall]

45. Y. Hirono, S. Yasui, K. Itakura and M. Nitta, "Non-Abelian statistics of vortices with multiple Majorana fermions," Phys. Rev. B 86, 014508 (2012). arXiv:1203.0173 [cond-mat.supr-con]

46. M. Eto, Y. Hirono, M. Nitta and S. Yasui, "Vortices and Other Topological Solitons in Dense Quark Matter," PTEP 2014, no. 1, 012D01 (2014). arXiv:1308.1535 [hep-ph]

47. S. Yasui, K. Itakura and M. Nitta, "Dirac returns: Non-Abelian statistics of vortices with Dirac fermions," Nucl. Phys. B 859, 261 (2012). arXiv:1109.2755 [cond-mat.supr-con]

48. S. Yasui, Y. Hirono, K. Itakura and M. Nitta, "Non-Abelian statistics of vortices with non-Abelian Dirac fermions," Phys. Rev. E 87, no. 5, 052142 (2013). arXiv:1204.1164 [cond-mat.supr-con]

49. M. Eto, J. Evslin, K. Konishi, G. Marmorini, M. Nitta, K. Ohashi, W. Vinci, N. Yokoi, On the moduli space of semilocal strings and lumps. Phys. Rev. D 76, 105002 (2007). arXiv:0704.2218 [hep-th] 